\title{
ESTADO DEL ARTE DE LA MÁSCARA LARÍNGEA PROSEAL
}

\section{INDICACIONES Y USOS ACTUALES}

Gisella Archbold De la Peña MD*, Andrés Fabricio Caballero Lozada MD*

\begin{abstract}
Resumen
En el año 2000 Archie Brain publicó la primera descripción de una nueva máscara laríngea que incorporó un segundo tubo lateral al de la vía aérea y cuyo extremo distal se localiza en la punta de la máscara. Tiene como finalidad separar el tracto respiratorio del digestivo, permitiendo así acceder al estómago con una sonda orogástrica para el drenaje del contenido al exterior. A este nuevo diseño se le conoce hoy como LMA ProSeal. Se describen las indicaciones actuales, la comparación con otros dispositivos de la vía aérea y sus complicaciones.
\end{abstract}

Palabras clave: máscara laríngea, ProSeal, dispositivos de vía aérea.

Abreviaturas: ML, máscara laríngea; MLP, máscara laríngea ProSeal.

\section{StATE OF THE ART - PROSEAL LARYNGEAL MASK AIRWAY INDICATIONS AND CURRENT USE}

\begin{abstract}
Archie Brain published the first description of the newly designed laryngeal mask in 2000 . It features a second tube parallel to the ventilation tube and its distal end located at the tip of the mask. It is designed to separate the respiratory tract from the gastrointestinal tract allowing a tube to be passed for aspiration of gastric contents. This new design is known as the ProSeal Laryngeal Mask Airway PLMA. Hereunder we describe current indications, comparison with other airway devices and complications.
\end{abstract}

Key words: laryngeal mask, ProSeal, airway devices 


\section{Introducción}

El manejo de la vía aérea representa múltiples retos para el anestesiólogo y dentro de la evolución de la especialidad se desarrollan nuevos dispositivos que van dirigidos a optimizar el manejo anestésico. Dentro del marco histórico aparece como primera opción la intubación endotraqueal, de la cual se encuentran diagramas desde 1443 en tráqueas de animales, pasando por la intubación en humanos mediante guía táctil a través de la laringe; después se desarrollaron dispositivos para hacer más efectivo su acceso. Es así como se desarrolló el primer laringoscopio por parte del Dr. Flagg en 1918, llegando hasta la Primera Guerra Mundial cuando se demostraron las ventajas de la intubación endotraqueal por parte de Magill y Roubothan..$^{1,2}$

La ML desde la invención por parte de Archie Brain en los años ochenta y su comercialización en 1988, ha ido ganando fuerza en la utilización, pues ha demostrado en múltiples estudios ventajas sobre el tubo endotraqueal como menor causante de traumatismo de la vía aérea, mayor facilidad de inserción, poca respuesta simpática durante su inserción y retiro, con escasas molestias en el posoperatorio..$^{3,4,5}$

La MLP fue diseñada con el objetivo de proporcionar una vía separada para permitir el flujo de contenido gástrico lejos de la glotis, siendo útil en los pacientes sin certeza de ayuno completo. Permite el acceso al estómago de sondas nasogástricas convencionales con cierre hermético alrededor de la glotis, disminución del flujo de gas inspirado al estómago y fijación más cómoda en la faringe, lo cual facilita el uso de ventilación mecánica y el diagnóstico de localización incorrecta de la MLP. ${ }^{6}$ Se ha mostrado que el riesgo de aspiración del contenido gástrico es menor gracias a la incorporación de un tubo de drenaje que en presencia de un inadecuado sello sobre la glotis, permite el drenaje del contenido gástrico protegiendo la vía aérea y permitiendo una ventilación con presión positiva más segura que con la ML convencional.

La indicación precisa para su uso actual comprende a los pacientes en que no se desea respuesta simpática cuando hay vía aérea difícil ni elevación de la presión intraocular, y en quiénes se desea evitar el laringoespasmo. Se contraindica en riesgo de broncoaspiración (es- tómagolleno, hernia hiatal, obesidad, embarazo, obstrucción intestinal), retraso en el vaciamiento gástrico y presencia de edema o fibrosis pulmonar. Además, ha ganado terreno en diferentes áreas como la reanimación cardiopulmonar, procedimientos quirúrgicos prolongados, vía aérea difícil, trauma cervical o incluso en posiciones quirúrgicas no convencionales como el decúbito prono. ${ }^{7-13}$

Al revisar la literatura se encuentran trabajos en los cuales la MLP ha sido usada en contextos diferentes a los previamente indicados; como resultado de estos estudios se ha estimadoun riesgosimilar de broncoaspiración al compararla con la ML convencional y el tubo endoraqueal, considerándolo como dispositivo protector de broncoaspiración, con menor tiempo en la intubación y mayor efectividad en el mantenimiento de la vía aérea. Por tales razones el propósito del artículo es describir el diseño y desarrollo de la MLP, sus indicaciones actuales, comparaciones con otros dispositivos y ventajas y complicaciones asociadas con su uso.

\section{Métodos}

Se realizó búsqueda y revisión de la literatura relacionada con el uso de la MLP en diferentes intervenciones, ya fueran quirúrgicas o no (por ejemplo, cuidado crítico o reanimación). Los artículos fueron encontrados en bases de datos (PUBMED, LILACS, LIBRERÍA COCHRANE), empleando las palabras clave ProSeal y ProSeal and PLMA (Figura 1). Se seleccionaron las referencias juntocon las halladas mediante procedimiento manual en revistas de anestesiología de enero 2000 hasta septiembre 2009 (Figura 2). La búsqueda identificó 18 ensayos clínicos controlados y otros estudios clínicos, quince reportes y series de casos, tres revisiones sistemáticas, seis cartas al editor y trece resúmenes.

Esta revisión se presenta en tres secciones:

1. El diseño de la máscara laríngea, aspectos técnicos del uso, detalles prácticos y rendimiento del dispositivo.

2. Comparaciones entre las MLP y otros dispositivos de la vía aérea.

3. Usos de la MLP y complicaciones de su uso. 


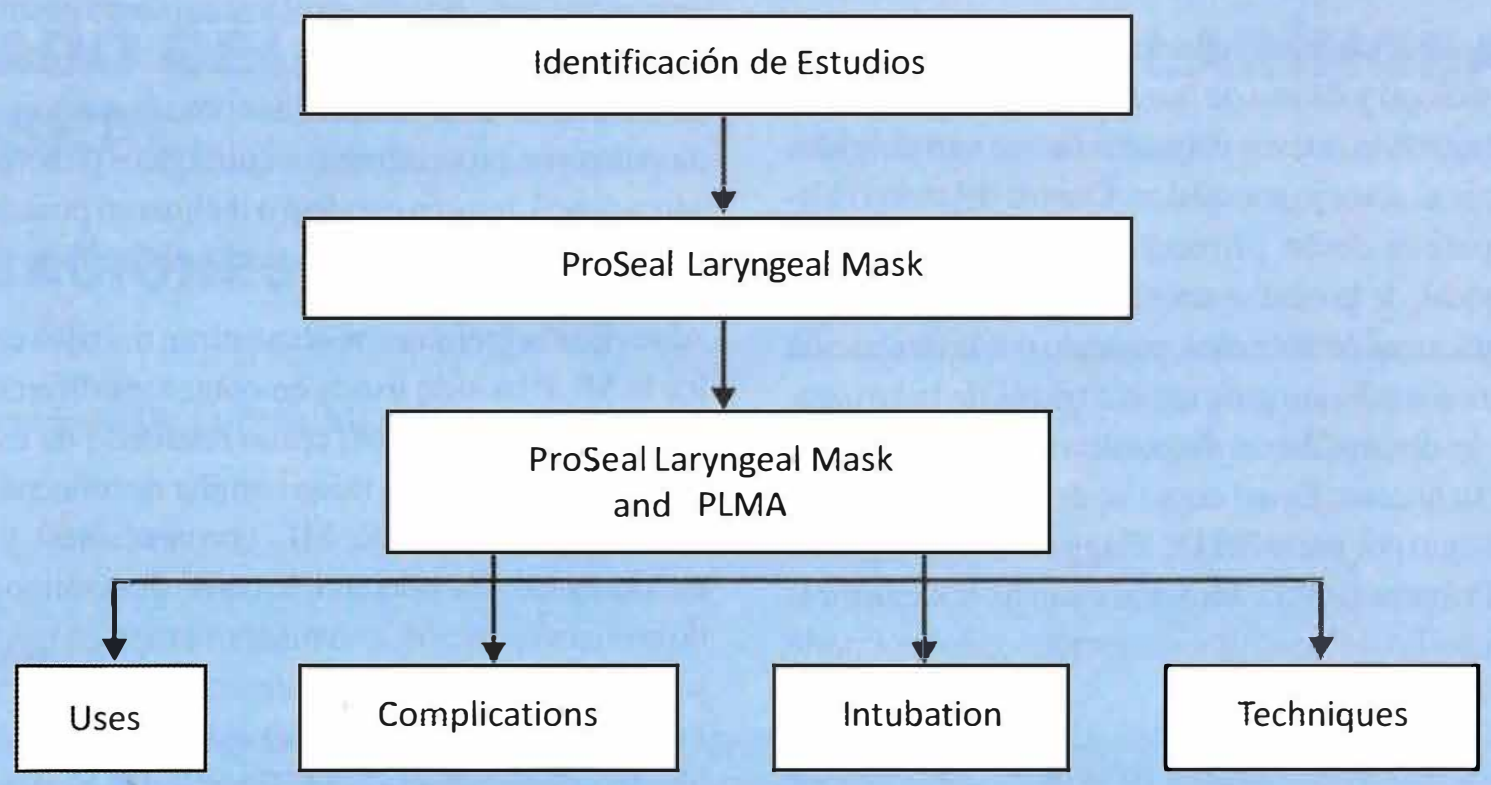

Figura I. Estrategia de búsqueda.

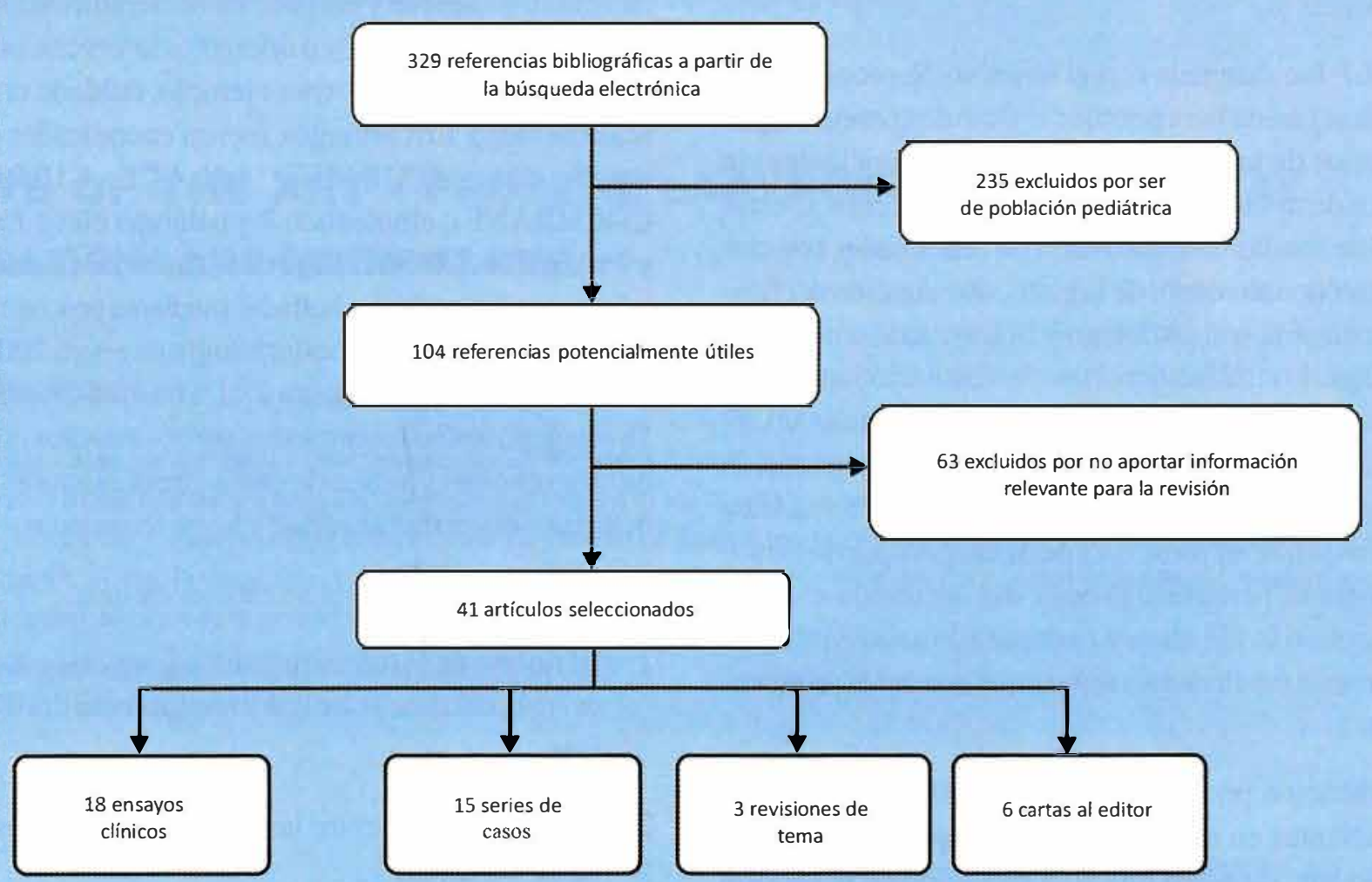

Figura 2. Estrategia de búsqueda. 


\section{Diseño de la MLP, aspectos técnicos, detalles prácticos y rendimiento del dispositivo}

La MLP es similar a la ML convencional; consiste en un tubocon una cámara profunda y un neumotaponador. El tubo aéreo es reforzado, pero con un calibre similar al de la ML convencional; las diferencias radican en (Figura 3, Tabla 1): 1) la concavidad es más profunda y amplia. 2) tiene una extensión posterior del balón de la máscara; 3 ) posee un tubo de drenaje que corre paralelo al tubo aéreo; 4) tiene unbloqueador de mordida de silicona y 5) posee un bolsillo anterior para colocar el introductor o el dedo durante la inserción (Figura 3).

El objetivo de estas modificaciones es: 1) evitar la inflación gástrica durante la ventilación; 2) menor necesidad de oclusión del esfínter esofágico superior por la punta de la máscara en la eventualidad de regurgitación gracias a la presencia de un tubo de drenaje y 3) ventaja en el paso de una sonda orogástrica. Estos cambios fueron diseñados para mejorar el sello en la vía aérea, además de permitir el rápido diagnóstico del desplazamiento de la máscara.
Cuando la máscara laríngea se coloca en posición correcta, el orificio cae sobre la glotis y el tubo de drenaje se apoya por detrás del cartílago cricoides en el origen del esófago. La vía aérea y el tubo de drenaje forman rutas ininterrumpidas hasta su salida en la boca. Esta separación funcional del tracto respiratorio y gastrointestinal es importante para entender las ventajas potenciales de la MLP sobre la convencional y los otros dispositivos supraglóticos. Se puede considerar que actúa como una laringe artificial, más que un tubo aéreo simple. ${ }^{3,4,6}$ La inserción es recomendable con la cabeza extendida y el cuello flexionado. Puede hacerse con o sin el introductor y cuando no se usa es similar a la ML convencional. ${ }^{14,15}$

El dedo índice se coloca en el bolsillo lo que hace más fácil la compresión lateral del cuerpo de la máscara y facilita el retiro del dedo. La MLP se presiona contra el paladar duro y avanza hasta la hipofaringe hasta encontrar resistencia; el dedo dentro del bolsillo se dirige hacia el occipucio, mientras se hace presión con la otra mano para que se coloque en el bolsillo de silicona ubicado en el extremo proximal entre el tubo de la vía aérea y el tubo de drenaje. La MLP se asemeja a la convencional y

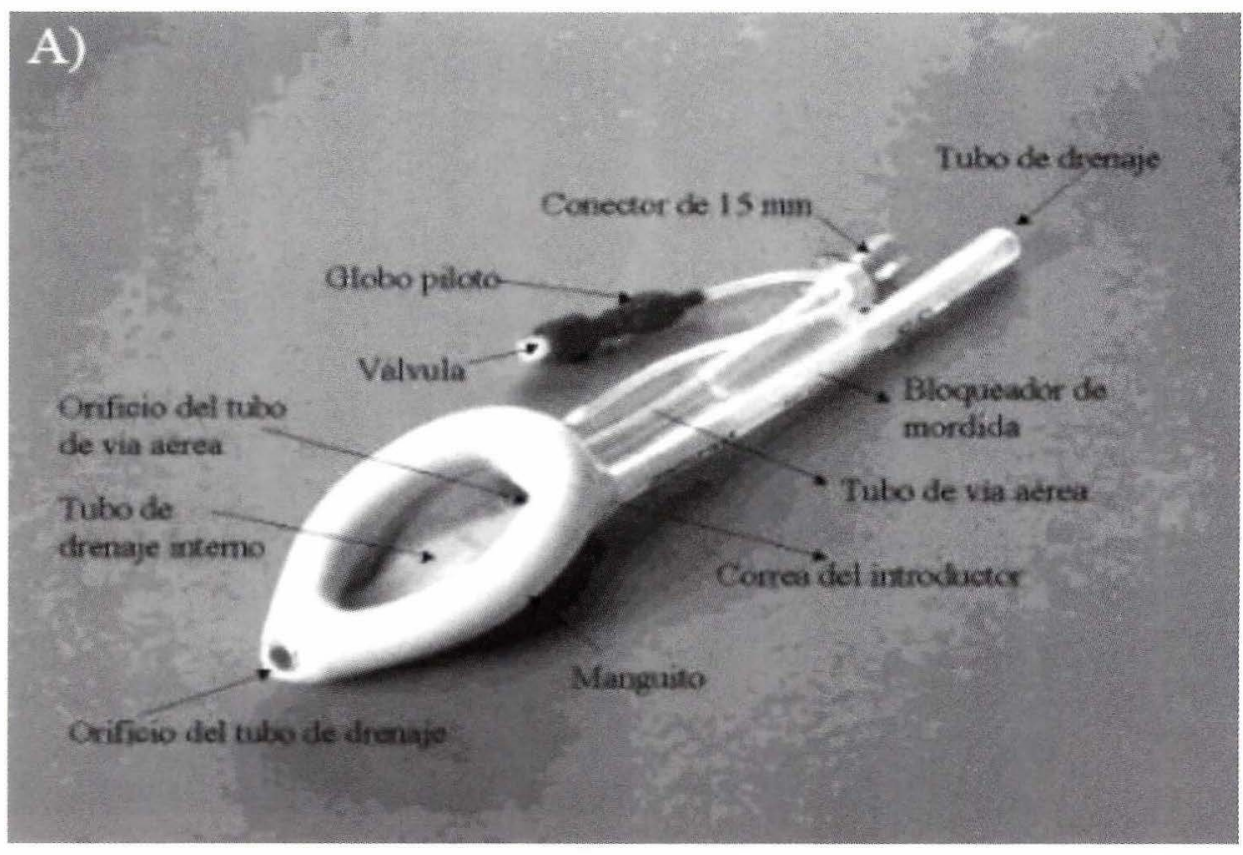

Figura 3. Diseño de la MLP. 
Tabla I. Dimensiones y detalles técnicos de la MLP

\begin{tabular}{|c|c|c|c|c|}
\hline Tamaño & Peso del paciente & $\begin{array}{c}\text { Máximo volumen } \\
\text { de inflación }(\mathrm{ml})\end{array}$ & $\begin{array}{c}\text { Diámetro máximo del } \\
\text { tubo orogástrico (fr) }\end{array}$ & $\begin{array}{c}\text { Distancia de la punta al } \\
\text { tubo de drenaje (cm) }\end{array}$ \\
\hline 1 I $/ 2$ & $5-10 \mathrm{~kg}$ & 7 & 10 & 18.2 \\
2 & $10-20 \mathrm{~kg}$ & 10 & 10 & 19.0 \\
$21 / 2$ & $20-30 \mathrm{~kg}$ & 14 & 14 & 23.0 \\
3 & $30-50 \mathrm{~kg}$ & 20 & 16 & 26.5 \\
4 & $50-70 \mathrm{~kg}$ & 30 & 16 & 27.5 \\
5 & $70-100 \mathrm{~kg}$ & 40 & 18 & 28.5 \\
\hline
\end{tabular}

su técnica de inserción es muy similar excepto por la posición de la cabeza que es de olfateo para la MLP y neutral para la otra. El manguito se coloca dentro de la boca, contra el paladar duro, avanzando con la guía en un arco suave hasta encontrar resistencia (Figura 4). Se debe retirar el introductor evitando una lesión dental.

Después de la inserción se infla el manguito hasta un límite de 60 ce de $\mathrm{H}_{2} 0$ para minimizar la presión sobre la mucosa faríngea. Ningún estudio ha reportado diferencias significativas en el éxito de inserción entre la técnica digital y la guiada por el introductor. ${ }^{16} \mathrm{Hwang}$ y cols. compararon la introducción rotacional del dispositivo con la técnica estándar y demostraron que con la primera hay escaso trauma de la faringe, que se evidencia por una menor incidencia de dolor en el cuello y sangrado de la mucosa $(\mathrm{p}<0.0001){ }^{17.18}$

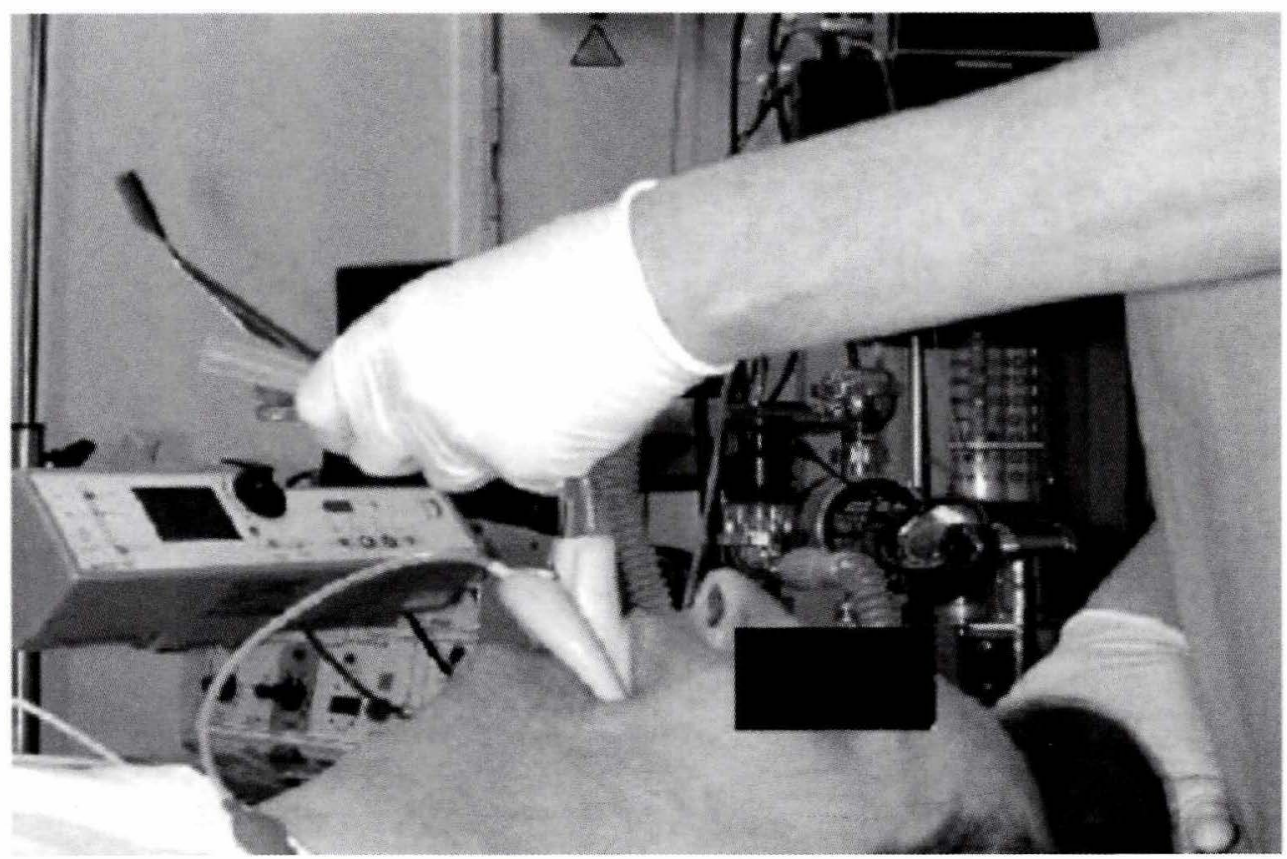

Figura 4. Técnica de inserción MLP. 


\section{Comparaciones entre la MLP y otros dispositivos de la vía aérea}

El uso de dispositivos supraglóticos en condiciones de presión intrabdominal requiere un excelente sello para separar el tracto respiratorio del digestivo y evitar el potencial riesgo de regurgitación. Cuando se compara la MLP con la clásica, Lu y cols. demostraron que la primera es superior y más efectiva durante la cirugía laparoscópica. El estudio multicéntrico realizado por Brimacombe en 384 pacientes programados para cirugía menor también lo evidenció. ${ }^{16.19-21}$

Comparada con el tubo laríngeo Roth y cols. demostraron que no existe diferencia en la inserción de los dispositivos, ambos permiten asegurar de manera rápida la vía aérea cuya presión después de la insuflación del peritoneo es similar, sin evidencia de dilatación gástrica, fuga del aire ni signos de regurgitación. Sin embargo, ellos observaron mayor incidencia de dolor en el cuello y disfagia posteriores a la remoción del dispositivo en los pacientes manejados con el tubo laríngeo ( $1 \%$ vs. $8 \%$ y $4 \%$ vs. $12 \%)^{22}$

Ensayos clínicos realizados en cadáveres han demostrado que la colocación de la MLP genera mayor presión sobre la mucosa laríngea que los otros dispositivos supraglóticos. ${ }^{23}$ Wahlen y col. en un estudio realizado en maniquíes con el objetivo de comparar la tasa de éxito y el tiempo de inserción de los distintos dispositivos de vía aérea entre diferentes grupos de personal de la salud, demostraron que los anestesiólogos realizaron la intubación orotraqueal más rápido $(\mathrm{p}<0.05)$. Pero los demás también fueron capaces de colocar la ML clásica, la MLP y el tubo laríngeo con una tasa de éxito mayor de $90 \%$ en el primer intento. Esto convierte a la MLP, al igual que los orros dispositivos supraglóticos, en herramientas útiles para el manejo de la vía aérea en situación de emergencia por personal no entrenado. ${ }^{24-27}$

\section{Indicaciones actuales}

\section{Cirugía general}

La MLP ha sidoutilizada como altemativa a la intubación ororaqueal en múltiples procedimientos quirúrgicos; sin embargo, la principal objeción para su utilización en ciru- gía prolongada y/o bajo ventilación con presión positiva o ambas es la falta de sello sobre la vía aérea y el consiguiente riesgo de insuflación gástrica y posible aspiración pulmonar. ${ }^{28}$

En su experiencia en 118 colecistectomías por laparoscopia, 72 con intubación orotraqueal y 46 con MLP, García-Aguado R. y col. demostraron que ésta última fue eficaz en la asistencia ventilatoria en todos los casos durante el procedimiento y requirió bajas dosis de relajantes neuromusculares en los momentos puntuales cuando se apreciaron fugas aéreas durante la ventilación. No hubo ningún caso de aspiración de contenido gástrico detectable en la clínica, ni de regurgitación hacia la hipofaringe. Se presentó uno solo de laringoespasmo en el grupo de tubo orotraqueal y las náuseas posoperatorias fueron de 23,9\% con MLP y $25,6 \%$ con orotraqueal. ${ }^{28}$ Se ha reportado también el uso de MLP en escenarios poco convencionales. En una serie de casos de 102 pacientes, Fabregat J. ${ }^{29}$ reportó, un sello adecuado en todos los casos, buena ventilación e inserción del dispositivo en el $96 \%$ de los pacientes, odinofagia en el 3\% e imposibilidad de colocación de la máscara en uno solo. ${ }^{26,30,31}$

\section{Cirugía laparoscópica ginecológica}

La mayoría de los procedimientos ginecológicos por patologías benignas son aptos para manejo quirúrgico por vía laparoscópica. Intervención muy poco invasiva que consiste en la insuflación de la cavidad abdominal con dióxido de carbono $\left(\mathrm{CO}_{2}\right)$ para crear un neumoperitoneo que permita al equipo quirúrgico obtener una visión clara del contenido abdominal. Al comparar la cirugía laparoscópica con la abierta se encuentran, entre otras, las siguientes ventajas: menor traumaa tejidos y baja morbilidad, mortalidad, estancia hospitalaria y reducido costo. Sin embargo, la cirugía laparoscópica se asocia con problemas mayores como complicaciones respiratorias, enfisema subcutáneo de $\mathrm{CO}_{2}$, neumotórax a tensión, embolismo gaseoso y lesiones no intencionales de órganos intrabdominales, por lo que es necesario asegurar la vía aérea y proporcionar una adecuada ventilación, lo que hace que la técnica preferida para este tipo de procedimientos sea la intubación orotraqueal con ventilación mecánica controlada. ${ }^{19}$ 
Desde su creación en los años ochenta, la máscara laríngea ha ocupado un lugar importante en la práctica clínica, en especial en procedimientos quirúrgicos de corta duración y bajo anestesia general con respiración espontánea. La ventaja que ofrece el uso de dispositivos supraglóticos como la MLP, radica en el hecho que puede ser fácil su inserción bajo una técnica ciega, sin requerir relajantes neuromusculares para la colocación o mantenimiento, lo que disminuye la incidencia de reacciones adversas a éstos, a los agentes de reversión de bloqueo neuromuscular o ambos, al igual que acorta el tiempo de extubación y por ende, también el de salas de cirugía. ${ }^{32}$ Además permite una ventilación adecuada con presión positiva, sello de la vía aérea y fugas mínimas. En comparación con el tubo orotraqueal la MLP muestra una baja incidencia de dolor en el cuello, asociado con menor estancia en la unidad de cuidado posanestésico, escaso requerimiento de medicación antiemética y analgésica, y disminución de la frecuencia de náuseas y vómito posoperatorios. ${ }^{33.34}$ Se ha demostrado que la MLP brinda un adecuado sello en condiciones de elevada presión intrabdominal, garantizando la separación del tracto digestivo y el respiratorio, con la ventaja de proporcionar un dispositivo de drenaje para contenido gástrico en caso de eventual regurgitación. ${ }^{35,36}$

\section{Unidad de cuidado intensivo}

Diversos estudios han evaluado la respuesta hemodinámica a la inserción de la MLP, comparada con el tubo orotraqueal. Se ha observado escasa respuesta hemodinámica durante la inserción así como durante el retiro en salas de cirugía. De igual manera, en el ámbito de la UCI la MLP ha mostrado resultados satisfactorios, sobre todo en pacientes con vía aérea difícil reconocida y con comorbilidades cardiovasculares importantes, o después de procedimientos neuroquirúrgicos programados para extubación diferida. Russo y col. ${ }^{11}$ demostraron en 40 pacientes programados para cirugía urológica o ginecológica, menos cambios hemodinámicas significativos con el uso de la MLP (presión arterial y frecuencia cardíaca con $\mathrm{p}<0.05$ ). La ventilación con MLP durante el traslado de salas de cirugía a la UCI se presentó sin eventos adversos significativos. , $^{9,37}$
Otra de las aplicaciones descritas de la MLP en UCI ha sido facilitar el destete ventilatorio en pacientes con extubación previa fallida por episodios de broncoespasmo a repetición, que pueden aumentar la necesidad de traqueostomía. En este sentido la ML clásica comparada con el tubo orotraqueal disminuye la incidencia de tos y complicaciones de la vía aérea, pero debido al sello inadecuado que esta proporciona, pueden ocurrir problemas con la ventilación controlada. La MLP se asocia con menor estimulación de la vía aérea durante la extubación y mejor adaptación a la ventilación mecánica. ${ }^{38}$

\section{Vía aérea difícil}

Gracias a las características técnicas de este dispositivo, se ha descrito su uso en la literatura internacional en casos de intubación difícil en la paciente obstétrica. Esta es la situación más importante que plantea la anestesia general en obstetricia y una de las principales causas de mortalidad materna. Los cambios anatómicos durante el embarazo (congestión mucosa, edema faringolaríngeo, hipertrofia mamaria) unidos a la maniobra de Sellick, dificultan la visión durante la laringoscopia. La incidencia de fallo en la intubación es más frecuente que en el resto de pacientes.

La experiencia del uso de la MLP durante la realización de cesárea es cada vez mayor. Múltiples autores lo han descrito como método de rescate ante una intubación difícil en cesáreas. Además, aparecen ya en la literatura grandes series de utilización de este dispositivo, mostrando su eficacia y seguridad. Por esto se considera una opción válida para el mantenimiento de la vía aérea. ${ }^{8,9,39}$ De igual manera, en un reporte de caso Cranshaw describió el uso de la MLP comoasistencia para la intubación con fibra óptica para vía aérea anticipada con laringoscopia directa. ${ }^{40,41}$

\section{MLP y decúbito prono}

El uso de la MLP en la posición decúbito prono es controversial. Aún así se considera que puede ser más seguro por el mejor sello y porque proporciona acceso al estómago. Brimancombe y col. reportaron su experiencia 
en 245 personas sanas y demostraron que su inserción fue fácil en todos los pacientes: 237 con ayuda digital y ocho con guía metálica. La ventilación fue adecuada en todos, solo tres presentaron obstrucción parcial de la vía aérea de fácil corrección y sin deterioro de la oxigenación, hipercapnia, desplazamiento ni insuflación gástrica. Debido a esto concluye quela inserción y mantenimiento de la anestesia con la MLP es factible por personal con entrenamiento adecuado. ${ }^{10.42}$ Se ha descrito también su utilidad en el rescate de la vía aérea en un paciente en posición decúbito prono con desplazamiento del tubo orotraqueal e imposibilidad de reintubación traqueal, logrando adecuados paráme ros ventilatorios para terminar con éxito el procedimientoquirúrgico. ${ }^{8}$

\section{Complicaciones asociadas con MLP}

La malposición de la MLP en el primer intento de inserción ocurre en 5 a $15 \%$, por lo que autores como O'Connor recomiendan comprobar siempre la posición. Se han descrito cinco tipos de malposiciones: 1) cuando la inserción es profunda, la punta distal quedará en la laringofaringe (7\%); 2) si la MLP toma una trayectoria anterior, la punta distal quedará en la entrada de la glotis $3 \%$; 3 ) cuando la inserción es defectuosa o se produce pérdida de la presión del manguito, la punta de la mascarilla puede doblarse sobre sí misma en la hipofaringe ( 3 a 4\%) lo cual puede evitarse con la utilización de técnicas de inserción guiada; 4) el descenso severo de la epiglotis $(<0,5 \%)$ suele ocurrir cuando se empuja hacia abajo por la punta de la MLP y cubre por completo la entrada de la glotis y 5) compresión de la glotis por la punta distal de la $\operatorname{MLP}(0,3 \%)$.

A través de la MLP es posible que ocurra distensión gástrica durante la ventilación con presión positiva a pesar de un adecuado sellado y una prueba de malposición negativa, pero suele ser baja incluso a altas presiones de la vía aérea. De hecho se ha descrito en sólo uno de 572 pacientes de nueve estudios. El diseño y las características de la MLP permiten esperar una reducción de la insuflación gástrica y aspiración pulmonar en comparación con la ML clásica. En diez cadáveres en los que se colocó la MLP y se dejó abierto el tubo de drenaje, proporcionó mejor protección de la vía aérea durante la regurgitación que la ML convencional, incluso con altas presiones en la vía. ${ }^{3,443-47}$ Cuando se requieren altas presiones de ventilación se observa fuga de gas con el uso de la MLP, pero menor que con la ML clásica. La fuga se dirige hacia la hipofaringe en el $95 \%$ de los casos, porcentaje similar a lo que ocurre con la convencional, pero el tubo de drenaje de la MLP la deriva del esófago haciendo menos probable la insuflación gástrica. Se han descrito quince reportes de casos de regurgitación sin aspiración con la MLP. En todos los casos el contenido gástrico, líquido o sólido se ha derivado por el tubo de drenaje sin alcanzar la vía aérea. ${ }^{23,44}$

La MLP no debe considerarse como totalmente segura cuando existe alto riesgo de regurgitación o aspiración. Debe tenerse en cuenta que la punta del tubo de drenaje debe estar en posición correcta para que funcione de manera apropiada. Se han publicado tres reportes de casos de aspiración real y dos probables, siendo la malposición del dispositivo la causa señalada en uno de ellos. La lesión de la mucosa se reconoce por la presencia de sangre en la MLP luego de su retiro, y varía entre 3 y $28 \%$ con una media de $10,2 \%$. La incidencia de dolor en el cuello varía de 2 a $49 \%$ con una media de $49 \%$ y una de las causas potenciales es la presión de la mucosa faríngea por la MLP. La relación entre el volumen del neumotaponador, las presiones sobre la mucosa y la presión de sello sobre la vía aérea fue estudiada en 32 pacientescon MLP y convencional, siendo más baja la presión sobre la mucosa con mejor presión de sello sobre la vía aérea al usar la primera. ${ }^{48-50}$

\section{Conclusiones}

La MLP es un dispositivo supraglótico que surgió como resultado de modificaciones en la ML convencional entre las cuales se destaca contar con un tubo de drenaje que separa el tracto digestivo del respiratorio, aumentar la protección contra la aspiración del contenido gástrico a nivel pulmonar y proporcionar un mejor sello sobre la vía aérea reduciendo el riesgo de insuflación gástrica. Su uso ha sido descrito en diferentes escenarios en la práctica clínica con un buen margen de seguridad y mínimos eventos adversos, incluso en situaciones que eran exclusivas para el tubo endotraqueal, como la cirugía en decúbito prono, procedimientos bajo laparoscopia, vía aérea difícil y soporte ventilatorio en UCI. Hasta el mo- 
mento existe evidencia de mínimas complicaciones derivadas de su uso, las cuales están relacionadas en esencia con mala técnica de inserción por falta de entrenamiento del operador, planos inadecuados de profundidad anestésica y la mala selección de pacientes para su colocación. Sin embargo, la gran mayoría de estudios cuentan con un número limitado de pacientes, lo que hace que los resultados no sean definitivos para su aplicación en la práctica clínica habitual de una manera siempre segura.

\section{Referencias}

1. Forsan d, Brain A. laryngeal mask airway. In: Hagberg CA, editor. Benumof's airway management. second ed. Texas: 2007. p. 476-501.

2. Gal T. Control de las vias respiratorias. In: Miller R, editor. Miller Anestesia. Sexta ed. San Francisco: 2009. p. 1617-52.

3. Cook TM, Lee G, Nolan JP. The ProSeal laryngeal mask airway: a review of the literature. Can J Anaesth 2005 Aug;52(7):739-60.

4. Brimacombe J, Keller C. The ProSeal laryngeal mask airway. Anesthesiol Clin North America 2002 Dec;20(4):871-91.

5. Tan BH, Chen EG, Liu EH. An evaluation of the laryngeal mask airway supreme in patients. Anaesth Intensive Care. 2010 May;38(3):550-4.

6. Izquierdo B, Lafuente N, Viu D, Ruiz R, Abengoechea JM, Ruiz J. [Proseal laryngeal mask]. Rev Esp Anestesiol Reanim 2006 Nov;53(9):556-65.

7. Gatward JJ, Thomas MJ, Nolan JP, Cook TM. Effect of chest compressions on the time taken to insert airway devices in a manikin. Br J Anaesth 2008 Mar;100(3):351-6.

8. Keller C, Brimacombe J, Lirk P, Puhringer F. Failed obstetric tracheal intubation and postoperative respiratory support with the ProSeal laryngeal mask airway. Anesth Analg 2004 May;98(5):1467-70, table.

9. Cook TM, Silsby J, Simpson TP. Airway rescue in acute upper airway obstruction using a ProSeal Laryngeal mask airway and an Aintree catheter: a review of the ProSeal Laryngeal mask airway in the management of the difficult airway. Anaesthesia 2005 Nov;60(11):1129-36.

10. Brimacombe J, Keller C. An unusual case of airway rescue in the prone position with the ProSeal laryngeal mask airway. Can J Anaesth 2005 Oct;52(8):884.

11. Russo SG, Goetze B, Troche S, Barwing J, Quintel M, Timmermann A. LMA-ProSeal for elective postoperative care on the intensive care unit: a prospective, randomized trial. Anesthesiology 2009 Jul;111(1):116-21.

12. Hwang NC. ProSeal Laryngeal Mask Airway in a myasthenia gravis patient for thymectomy. Anaesth Intensive Care 2007 Apr;35(2):310.

13. Tanaka TT, Furutani HF, Harioka TH. Anaesthetic management of a patient with relapsing polychondritis undergoing laparoscopic surgery. Anaesth Intensive Care 2006 Jun;34(3):372-4.
14. Chen HS, Liu PH, Chung KC, Hung KC. Use of the Rusch Flexi-Slip stylet for patients with difficult insertion of the ProSeal laryngeal mask aurway. Acta Anaesthesiol Taiwan. 2010 Mar;48(1):37-40.

15. Jeon YT; Na HS,. Park SH, Oh AY, Park HP, Yun MJ, Kim JH, Hwang JW. insertion of the ProSeal laryngeal mask airway is more successful with the 90 degrees rotation technique. Can J. Anaesth. 2010 Mar;57(3):211-5.

16. Bercker S, Schmidbauer W, Volk T, Bogusch G, Bubser HP, Hensel M, et al. A comparison of seal in seven supraglottic airway devices using a cadaver model of elevated esophageal pressure. Anesth Analg. 2008 Feb; 106(2):4458 , table.

17. Hwang JW, Park HP, Lim YJ, Do SH, Lee SC, Jeon YT. Comparasion oftwo insertion techniques ofProSeal laryngeal mask airway: standard versus 90-degree rotation. Anesthesiology. 2009 Apr; 110(4):905-7.

18. Wessels CC. Hazard due to proseal tear. Anaesth Intensive Care. 2010 Jan;38(1):212.

19. Piper SN, Triem JG, Rohm KD, Maleck WH, Schollhom TA, Boldt J. (ProSeal-laryngeal mask versus endotracheal intubation in patients undergoing gynaecologic laparascopy). Anasthesiol Intensivmed Notfallmed Schmerzther. 2004 Mar;39(3):132-7.

20. Brimacombe J, Keller C, Fullekrug B, Agro F, Rosenblatt W, Dierdorf SF, et al. A multicenter study comparing the ProSeal and Classic laryngeal mask airway in anesthetized, nonparalyzed patients. Anesthesiology 2002 Feb;96(2):289-95.

21. Brimacombe J, Keller C. The ProSeal laryngeal mask airway: A randomized, crossover study with the standard laryngeal mask airway in paralyzed, anesthetized patients. Anesthesiology $2000 \mathrm{Jul} ; 93(1): 104-9$.

22. Roth H, Genzwuerker HV, Rothhaas A, Finteis T, Schmeck J. The ProSeal laryngeal mask airway and the laryngeal tube Suction for ventilation in gynaecological patients undergoing laparoscopie surgery. Eur J Anaesthesiol. 2005Feb;22(2):117-22.

23. Ulrich-Pur H, Hrska F, Krafft P, Friehs H, Wulkersdorfer B, Kostler WJ, et al. Comparison of mucosal pressures induced by cuffs of different airway devices. Anesthesiology.2006 May:104(5):933-8.

24. Wahlen BM, Roewer N, Lange M, Kranke P. Tracheal intubation and altemative airway management devices used by healthcare professionals with different level of pre-existing skills: a manikin study. Anaesthesia 2009 May;64(5):549-54.

25. Pay LL, Lim Y. Comparison of the modified Airway Management Device with theProseal laryngeal mask airway in patients undergoing gynaecological procedures. Eur J Anaesthesiol 2006 Jan;23(1):71-5.

26. Lu PP, Brimacombe J, Yang C, Shyr M. ProSeal versus the Classic laryngeal mask airway for positive pressure ventilation during laparoscopic cholecystectomy.Br J Anaesth. 2002 Jun;88/6):824-7.

27. Cavus E, Deitmer W, Francksen H, Serocki G, Bein B, Scholz J, Doerges V. Larungeal tube S II, ProSeal laryngeal mask, and Easy Tube during elective surgery: a randomized controlled comparison with the endotracheal tube in nontrained professionals. Eur J Anaesthesiol. 2009 Sep;26(9):730-5.

28. Garcia-Aguado R, Vivo BM, Zaragoza FC, Garcia Solbes JM. [ProSeal laryngeal mask for laparoscopic cholecystectomy]. Rev Esp Anestesiol Reanim 2003 Jan;50(1):55-7.

29. Hohlrieder M, Brimacombe J, Eschertzhuber S, Ulmer H, Keller C. A study of airway management using the ProSeal LMA laryngeal mask airway compared 
with the tracheal tube on postoperative analgesia requirements following gynaecological laparoscopic surgery. Anaesthesia 2007 Sep;62(9):913-8.

30. Fabregat J, De AC, Brimacombe J. Successful use of the classic LMA after failed use of the ProSeal LMA. Anaesth Intensive Care 2006 Apr;34(2):283.

31. Maltby JR, Beriault MT, Watson NC, Liepert D, Fick GH, The LMAProSeal is an effective alternative to tracheal intubation for laparoscopic cholecystectomy. Can J Anaesth. 2002 Oct;49(8):857-62.

32. Freo U, Carron M, Micaglio M, Ori C. ProSeal laryngeal mask airway for laparoscopic gastric banding in a myasthenic, morbidly obese patient. $\mathrm{Br} \mathrm{J}$ Anaesth 2007 Dec;99(6):921-2.

33. Hohlrieder M, Brimacombe J, von GA, Keller C. Postoperative nausea, vomiting, airway morbidity, and analgesic requirements are lower for the ProSeal laryngeal mask airway than the tracheal tube in females undergoing breast and gynaecological surgery. Br J Anaesth 2007 Oct;99(4):576-80.

34. Pandey R, Garg R, Nath MP, Rajan S, Punj J, Darlong V, Chandralekha. Eisenmenger's syndrome in pregnancy: use of proseal laryngeal mask airway (PLMA) an epidural analgesia for elective cesarean section. Acta Anaesthesiol Taiwan. 2009 Dec;47(4):204-7,

35. Cook TM, Gibbison B. Analysis of 1000 consecutive uses of the ProSeal laryngeal mask airway by one anaesthetist at a district general hospital. $\mathrm{Br} \mathrm{J}$ Anaesth 2007 Sep;99(3):436-9.

36. Maltby JR, Beriault MT, Watson NC, Liepert DJ, Fick GH. LMA-Classic and LMA-ProSeal are effective alternatives to endotracheal intubation for gynecologic laparoscopy. Can J Anaesth 2003 Jan;50(1):71-7.

37. DahabaAA, Prax N, Gaube W, Gries M, Rehak PH, Metzler H. Haemodynamic and catecholamine stress responses to the Laryngeal Tube-Suction Airway and the Proseal Laryngeal Mask Airway. Anaesthesia 2006 Apr;61(4):330-4

38. Laver S, McKinstry C, Craft TM, Cook TM. Use of the ProSeal LMA in the ICU to facilitate weaning from controlled ventilation in patients with severe two episodic bronchospasm. Eur J Anaesthesiol 2006 Nov;23(11):977-8.

39. de Blas GM, Martinez JF, Gredilla E, Gilsanz F. [Management of difficult intubation with a ProSeal-type laryngeal mask for an emergency cesarean section]. Rev Esp Anestesiol Reanim 2007 Mar;54(3):199-200.
40. Gongireddy VK, Das S. Use of LMA ProSeal as a dedicated airway for fibreoptic-guided racheal intubation. Anaesthesia $2006 \mathrm{Jul}$;61(7):715-6.

41. Cranshaw J, Shewry E. Use of ProSeal laryngeal mask airway as a dedicated airway for fibreoptic-guided tracheal intubation. Anaesthesia 2006 Feb;61(2):199-201.

42. Brimacombe JR, Wenzel V, Keller C. The proseal laryngeal mask airway in prone patients: a retrospective audit of 245 patients. Anaesth Intensive Care 2007 Apr;35(2):222-5.

43. Brimacombe J, Keller C. The ProSeal laryngeal mask airway may prevent of hiccup-related aspiration. Eur J Anaesthesiol 2005 Aug;22(8):636-8.

44. Keller C, Brimacombe J, Kleinsasser A, Loeckinger A. Does the ProSeal laryngeal mask airway prevent aspiration of regurgitated fluid? Anesth Analg 2000 Oct;91(4):1017-20.

45. Keller C, Brimacombe J, Hoermann C, Loeckinger A, Kleinsasser A. Pressure support ventilation with the ProSeal laryngeal mask airway. A comparison of sevoflurane, isoflurane and propofol. Eur J Anaesthesiol 2005 Aug;22(8):630-3

46. Brimacombe J, Keller C, Berry A. Gastric insufflation with the ProSeal laryngeal mask. AnesthAnalg 2001 Jun;92(6):1614-5.

47. Brimacombe J, Keller C. Aspiration of gastric contents during use of a ProSeal laryngeal mask airway secondary tounidentifiedfoldover malposition. AnesthAnalg 2003 Oct;97(4):1192-4, table.

48. Stix MS, O'Connor CJ, Jr., Valade DR. The ProSeal LMA does not cause laryngeal edema. Can J Anaesth 2006 Sep;53(9):961-3.

49. Moret GA, Fabregat LJ. [Ventilation and airway seal failure with a ProSeal laryngeal mask secondary to arytenoid edema caused by a nasogastric tube]. Rev Esp Anestesiol Reanim 2006 Mar;53(3):197-8.

50. Chin KJ, Chee VW. Laryngeal edema associated with the ProSeal laryngeal mask airway in upper respiratory tract infection. Can J. Anaesth. 2006 Apr;53(4):389-92. 